\title{
Special Issue of BICS 2016
}

\author{
Cheng-Lin Liu ${ }^{1} \cdot$ Amir Hussain $^{2} \cdot$ Bin Luo $^{3} \cdot$ Kay Chen $\operatorname{Tan}^{4} \cdot$ Yi Zeng ${ }^{1} \cdot$ Zhaoxiang Zhang $^{1}$
}

Published online: 2 April 2018

(C) Springer Science+Business Media, LLC, part of Springer Nature 2018

Brain-inspired cognitive models and algorithms are important components driving artificial intelligence (AI). Deep neural networks are currently considered the most effective models to yield high perception and inference performance by learning from big data. However they manifest inferior generalization, robustness, interpretability, and adaptability when compared to the human brain. Despite neural circuits and cognition mechanisms of the brain having many unknowns, they continue to inspire AI in different ways. The International Conference on Brain Inspired Cognitive System (BICS) has been organized since 2004 to stimulate interdisciplinary research and exchanges in brain-inspired cognitive systems and applications in diverse fields. The 8th International Conference on Brain Inspired Cognitive System (BICS 2016) was held in Beijing, China, November 28-30, 2016. This special issue aims to report new advances since BICS 2016, by including expanded versions of selected conference papers and also new contributions.

Until April 20, 2017, the special issue received 18 submissions, most of which were expanded versions of BICS 2016 conference papers, along with a few new submissions. Following a rigorous peer review process, nine papers were accepted for publication in this special issue. The nine papers present contributions in brain information processing, braininspired cognitive models, and algorithms for decision, learning, vision, and applications.

In "Anatomical Pattern Analysis for Decoding Visual Stimuli in Human Brains," Yousefnezhad and Zhang propose Anatomical Pattern Analysis (APA) for decoding visual stimuli in the human brain. This framework develops a novel

Cheng-Lin Liu

liucl@nlpria.ac.cn

1 Institute of Automation of Chinese Academy of Sciences, Beijing, China

2 University of Stirling, Stirling, Scotland

3 Anhui University, Hefei, China

4 City University of Hong Kong, Kowloon Tong, Hong Kong, China anatomical feature extraction method and a new imbalance AdaBoost algorithm for binary classification. Further, it utilizes an Error-Correcting Output Codes (ECOC) method for multiclass prediction. APA can automatically detect active regions for each category of the visual stimuli. Moreover, it enables us to combine homogeneous datasets for applying advanced classification. Experiments on four visual categories in fMRI data demonstrate the effectiveness of the proposed method.

In "A Brain-inspired Decision Making Model based on Top-down Biasing of Prefrontal Cortex to Basal Ganglia and Its Application in Autonomous UAV Explorations," Zhao et al. propose a general computational model, termed the prefrontal cortex-basal ganglia (PFC-BG) algorithm, for decision making. The proposed model is inspired by the biological reinforcement learning pathway and mechanisms from two perspectives: (1) It maintains the contextual reward information in working memory to yield the top-down biasing effect on reinforcement learning in basal ganglia; (2) Dopamine signals continuously update reward-relevant information for both basal ganglia and working memory in the prefrontal cortex. The proposed model separates the continuous states into smaller distinguishable states and introduces continuous reward functions for each state to obtain reward information at different times. Applications of the proposed model to UAV decision making experiments, such as avoiding obstacles and flying through a window and door, the experiments demonstrated the effectiveness of the model in respect to accuracy and fast decision making.

In "Towards Robot Self-consciousness (II): Brain-inspired Robot Bodily Self Model for Self-Recognition," Zeng et al. propose a brain-inspired robot bodily self-model based on extensions to the primate mirror neuron system and apply it to a humanoid robot for self-recognition. In this model, the robot firstly learns the correlations between self-generated actions and visual feedbacks in motion by learning with Spike Timing Dependent Plasticity (STDP), and then learns the appearance of a body part with the expectation that the visual feedback is consistent with its motion. Based on this model, the robot uses multisensory integration to learn its own body in real world and in mirror. Thus, it can distinguish itself from 
others. In a mirror test setting with three robots with the same appearance, with the proposed model, each of them could recognize itself in the mirror after the robots made random movements at the same time.

The next two papers address brain-inspired learning. In "End-to-End Lifelong Learning: a Framework to Achieve Plasticities of both the Feature and Classifier Constructions," Hao et al. propose an end-to-end lifelong learning framework to achieve plasticities of both the feature and classifier constructions in incremental learning. The proposed model is mainly comprised of three parts: Gabor filters followed by max pooling layer offering shift and scale tolerance to input samples, incremental unsupervised feature extraction and incremental SVM trying to achieve plasticities of both the feature learning and classifier construction. The plasticity in this model does not rely on the back propagation (BP) process and does not need huge parameters. The resulting incremental models, IncPCANet and IncKmeansNet, have achieved better results than PCANet and KmeansNet in experiments, and show promising plasticity of feature extraction and classifier construction when the distribution of data changes.

In "Incremental Adaptive Learning Vector Quantization for Character Recognition with Continuous Style Adaptation," Shen and Liu propose an incremental learning vector quantization (LVQ) algorithm for character recognition considering writing style adaptation in time sequence. They reasonably assume that adjacent characters from the same font or the same writer share the same style in a short period while style variation occurs in a long period. Based on this, the proposed continuous incremental adaptive LVQ (CIALVQ) method incrementally learns a self-adaptive style transfer matrix for mapping input patterns from style-conscious space onto style-free space. Following style transformation, the problem is reduced to a common character recognition task using the incremental LVQ (ILVQ) classifier, which can be learned in two modes: supervised incremental learning and active incremental learning. In the latter mode, samples receiving low confidence from the classifier are requested class labels. In experiments on NIST hand-printed data sets, the classification performance of CIALVQ was evaluated in two scenarios: interleaved test-then-train and style-specific classification. The results show that local style consistency improves the accuracies of both test scenarios, as well as for both supervised and active incremental learning modes.

The next four papers address visual information processing and applications of brain-inspired systems. In "Learning Optimal Seeds for Ranking Saliency," Wang et al. present a new optimal seeds method to obtain a saliency map from an image. First, they evaluate the salience value of each region using global contrast based spatial and color features. Second, the salience values of the first stage are used to optimize the background and foreground queries (seeds), and meanwhile, boundary cues from a hierarchical graph are taken to optimize background seeds. Then, saliency measures are derived from the classical manifold ranking after obtaining optimal seeds. The final saliency map is obtained by combining the saliency results of the two stages. When comparing with nine state-ofthe-art methods on five public datasets, the proposed method was shown to be able to handle complex images with different details, and produce more accurate saliency maps.

In "Compressing and Accelerating Neural Network for Facial Points Localization," Zeng et al. propose a method to compress and accelerate large deep neural network (DNN) models while maintaining performance. It includes three steps: (1) Importance-based pruning: introducing weights correlations to find and prune unimportant neurons or connections; (2) Product quantization: to better enforce weights shared than scalar quantization; (3) Network retraining: retraining the network iteratively after compressing one layer at a time. Further, all pooling layers are removed and the strides of their neighbor convolutional layers are increased to accelerate the network simultaneously. Experimental results of compressing a VGG-like model demonstrate the effectiveness of the method, which achieves $26 \times$ compression and $4 \times$ acceleration with a small root mean squared error (RMSE).

In "Hierarchical Convolutional Neural Networks for EEGbased Emotion Recognition," Li et al. propose to use hierarchical convolutional neural network (HCNN) for classifying the positive, neutral and negative emotion states in EEG data in order to overcome overfitting. They organize differential entropy features from different channels as two-dimensional maps to train the HCNNs, and use stacked autoencoder (SAE), SVM, and KNN as competing methods. Experimental results show that the HCNN yields the highest accuracy, and SAE is slightly inferior. Both of them demonstrate absolute advantages over traditional shallow models including SVM and KNN. The results also confirm that the high-frequency wave bands Beta and Gamma are the most suitable bands for emotion reading.

In "A Primal Neural Network for Online EqualityConstrained Quadratic Programming," Chen and Zhang propose an improved primal recurrent neural network and its electronic implementation for solving online quality-constrained quadratic programming problems. Compared to existing recurrent networks, such as GradientNet and ZhangNet, the proposed network can theoretically guarantee superior global exponential convergence. The network is also analyzed under a large model implementation error, with the upper bound of stead-state solution error estimated. Simulation results verify the theoretical analysis and demonstrate the effectiveness of the proposed model for online equality-constrained quadratic programming.

We thank all authors who submitted their invaluable works to this special issue and all the reviewers for their insightful comments. We specially thank Prof. Amir Hussain, the Editorin-Chief of Cognitive Computation for giving us the opportunity of guest editing this special issue. We also thank the journal manager Vincent Salvo for his support and assistance. 